\title{
Exercise-associated hyponatraemia after a marathon: case series
}

\author{
A M Goudie ${ }^{1} \quad$ D S Tunstall-Pedoe ${ }^{2} \quad$ M Kerins $^{3} \quad$ J Terris ${ }^{4}$
}

\section{SUMMARY}

Objectives To review the presentation, treatment and response of those runners from the London Marathon who presented to St Thomas' Hospital with exercise induced hyponatraemia.

Design Observational case series.

Setting St Thomas' Hospital, a tertiary hospital situated near the finish line of the 2003 London Marathon.

Participants All runners who presented to St Thomas' Hospital on the day of the 2003 London Marathon with altered mental state whose serum sodium concentration was less than $135 \mathrm{mmol} / \mathrm{L}$.

Main outcome measures Presenting symptoms, volume and type of fluids administered and response to treatment (biochemical and clinical).

Results Fourteen patients were diagnosed with exercise associated hyponatraemia with serum sodium concentrations ranging from 116 to $133 \mathrm{mmo} / \mathrm{L}$. Eleven presented with confusion. There were long delays between the finish time and presentation time for some runners. Anecdotal descriptions suggested some runners finished the race with normal mental state then became confused. There was no correlation between running time and serum sodium level. All patients received 0.9\% saline and six received 1.8\% saline. Despite this, some patients demonstrated falls in serum sodium concentrations. Thirteen to fourteen patients were symptomatically well the following morning, with the remaining patient significantly improved.

Conclusion Presentation of exercise associated hyponatraemia may be delayed. Optimal treatment is controversial, but the use of isotonic saline may not result in rises of serum sodium and we would suggest the early use of hypertonic fluids in symptomatic patients.
${ }^{1}$ Emergency Consultant, Swan District Hospital, Perth, Australia; ${ }^{2}$ Medical Director London Marathon, Cardiac Department, Homerton Hospital, London; ${ }^{3}$ Emergency Consultant, King's College Hospital, London; ${ }^{4}$ Emergency Consultant, St Thomas' Hospital, London, UK

Correspondence to: A M Goudie

E-mail: adrian.goudie@health.wa.gov.au

\section{INTRODUCTION}

Exercise-associated hyponatraemia (EAH) is a cause of collapse and confusion after prolonged exercise such as marathons and triathlons. ${ }^{1-3}$ Initially attributed to salt loss, it is now thought to be predominantly due to excessive ingestion of hypotonic fluids during and after the period of exertion and its associated suppression of diuresis. ${ }^{1-5}$ Although many competitors who develop hyponatraemia may remain minimally symptomatic or asymptomatic, severe complications including hyponatraemic encephalopathy, pulmonary oedema and death have been described. ${ }^{1,4,6}$ Individual risk factors include: female gender; use of non-steroidal anti-inflammatory drugs; slow running speed'; and excessive fluid ingestion. 5,7,8 Weather conditions and advice given to runners on drinking also have a profound effect on the overall incidence. ${ }^{1,9}$

Hyponatraemic encephalopathy is a potentially life threatening condition, with cerebral oedema causing vomiting, headache, confusion, coma, seizures and pulmonary oedema. ${ }^{1,4,6}$ It is most commonly associated with the syndrome of inappropriate anti-diuretic hormone secretion (SIADH) or in the post-operative setting. Less frequent causes include psychogenic polydypsia or other forms of excessive fluid consumption. ${ }^{10-12}$ 'Ecstasy' (3,4methylenedioxymethamphetamine) has become a relatively frequent cause in young people by producing a state of SIADH in the setting of strong encouragement of free water ingestion (to prevent hyperthermia) ${ }^{13}$. Treatment of hyponatraemic encephalopathy is controversial, with a need to rapidly correct the hyponatraemia being balanced by fear of too rapid or overcorrection, ${ }^{10}$ as this has been linked to cerebral demyelination. What should be considered a safe rate of correction is controversial. In the setting of seizures, rapid correction (7-10 mmol/L over $10 \mathrm{~min}$ ) using sodium loads in the order of hundreds of millimoles has been advocated, 4,14 although others advise slower rates of correction. ${ }^{10,11}$

In the setting of EAH, treatment recommendations are similarly variable, with regimes ranging from fluid restriction, $0.9 \%$ saline and hypertonic saline being described. A recent Consensus Conference has advised $100 \mathrm{~mL}$ of $3 \%$ saline repeated hourly. ${ }^{1,6,15}$ Rapid and complete recovery is usual with the return of post exercise diuresis and resolution of hyponatraemia. $1,6,7,9,15$ 
Ranging in age from 18 to 92 years, 32563 runners completed the 2003 London Marathon, of whom 24\% were women. Water stations were located every mile from 3 to 25 miles and Lucozade sport (a sports drink containing $22 \mathrm{mmol} / \mathrm{L}$ sodium $)^{16}$ was available at the $5,10,15,19$ and 23 mile stations (DS Tunstall-Pedoe, personal communication, 2004). We have previously described the 40 runners from the 2003 London Marathon who presented to the Emergency Department of St Thomas' Hospital - a tertiary level hospital situated near the finishing linewhich included one of the largest described cohorts of runners with EAH. ${ }^{2}$ The place of intravenous hypertonic saline, which may not be readily available in many hospitals and has been linked to central pontine myelinolysis ${ }^{10}$ when used in non-acute hyponatraemia, was assessed in this study.

\section{METHODS}

A record of the hospital record number and race number was made on the day of the event of those patients presenting to the Emergency Department of St Thomas' Hospital who had participated in the London Marathon. The hospital records were correlated with the St John Ambulance, London Ambulance Service and London Marathon hospital transfer data to ensure that all eligible runners had been included. Data was extracted retrospectively from the notes by one of the authors (MK) and cross-checked for accuracy by one of the other authors (AG).

According to a previously determined treatment algorithm, all patients who presented with collapse or altered mental state had a rectal temperature and capillary blood glucose measured. Any patient with altered mental state at presentation to the emergency department had a serum sodium concentration taken. Hyponatraemia was defined as a serum sodium concentration below $135 \mathrm{mmol} / \mathrm{L}$; $0.9 \%$ saline was used as the initial fluid of choice for those runners assessed as being dehydrated, and for those with symptomatic hyponatraemia. Those hyponatraemic runners who did not improve as expected, or who deteriorated, were administered hypertonic saline (1.8\%).

Statistical analysis was performed using Excel X for Mac (Microsoft Corporation 2001).

\section{RESULTS}

Fourteen patients were diagnosed with EAH, with serum sodium concentrations ranging from 116 to $133 \mathrm{mmol} / \mathrm{L}$ (Table 1). Eleven patients presented with confusion which was often marked (disorientated to time, place and person or making incomprehensible noises) and associated with agitation. Glasgow Coma Scores ranged from 11-15. The patient with the lowest sodium concentration (patient 3) one on arrival at the emergency department. There was a previous history of two seizures, but no diagnosis of epilepsy had been made. Both seizures stopped spontaneously. Three of the 14 patients had episodes of collapse and seven vomited.

All of the runners who presented with altered mental state had either low sodium or elevated temperature. ${ }^{2}$ In the hyperthermic group, serum sodium concentrations ranged from 136 to $143 \mathrm{mmol} / \mathrm{L}$.

Seven of the 14 patients with EAH were women, compared to $24 \%$ of race finishers $(P<0.03)$. All 14 had completed the marathon, with times ranging from $2 \mathrm{~h}$ $59 \mathrm{~min} 59 \mathrm{~s}$ to $5 \mathrm{~h} 38 \mathrm{~min} 35 \mathrm{~s}$. The proportion of those runners who suffered EAH who finished under $4 \mathrm{~h}$ was not statistically different from total finishers $(21 \%$ versus $34 \%$, $P>0.3)$. There was no correlation between finishing time and serum sodium concentration (Figure 1, $R=-0.02$ ).

Significant delays were noted between the finishing time and the time of presentation to the emergency department (mean delay 243, range 132-391 min). While it was not possible to definitely determine the time of symptom onset, collateral history was available in some cases, and some runners were described as finishing with apparently normal mental state, only to become confused later.

Two patients underwent cranial computerized tomography. Patient 5 was confused and had evidence of a head injury (scalp abrasion). Patient 10 had an erroneous initial serum sodium reading taken using a venous gas sample with unbalanced sodium heparin in the accident and emergency department. This demonstrated only mild hyponatraemia, inconsistent with the clinical condition. The formal laboratory result demonstrated more profound hyponatraemia (in keeping with the clinical condition). The blood gas analyser subsequently reported a calibration error for the sodium electrode. Both cranial computerized tomography results were normal.

All patients received $0.9 \%$ saline and six received 1.8\% saline, in amounts ranging from $250 \mathrm{~mL}$ to $1000 \mathrm{~mL}$. Details of fluid administration and changes in serum sodium during the first $4 \mathrm{~h}$ for those administered hypertonic saline are presented in Table 2. Patient 1 demonstrated a transient fall in her serum sodium at $1 \mathrm{~h}(122 \mathrm{mmol} / \mathrm{L}$ to $121 \mathrm{mmol} / \mathrm{L})$ despite being administered $500 \mathrm{~mL} / \mathrm{h}$ of $0.9 \%$ saline at the time, and patient 8 demonstrated a fall over $4 \mathrm{~h}$ despite $2000 \mathrm{~mL}$ of $0.9 \%$ saline and $500 \mathrm{~mL}$ of $1.8 \%$ saline.

Thirteen of the fourteen patients were symptomatically well the following morning. The remaining patient (patient 1) had improved markedly, but still had subtle memory impairment. She had made a complete recovery by the following morning. Most patients had no recall of running the marathon.

Only one patient was able to give an estimate of his fluid ingestion, of approximately $13 \mathrm{~L}$ consumed during the $5 \mathrm{~h}$ 


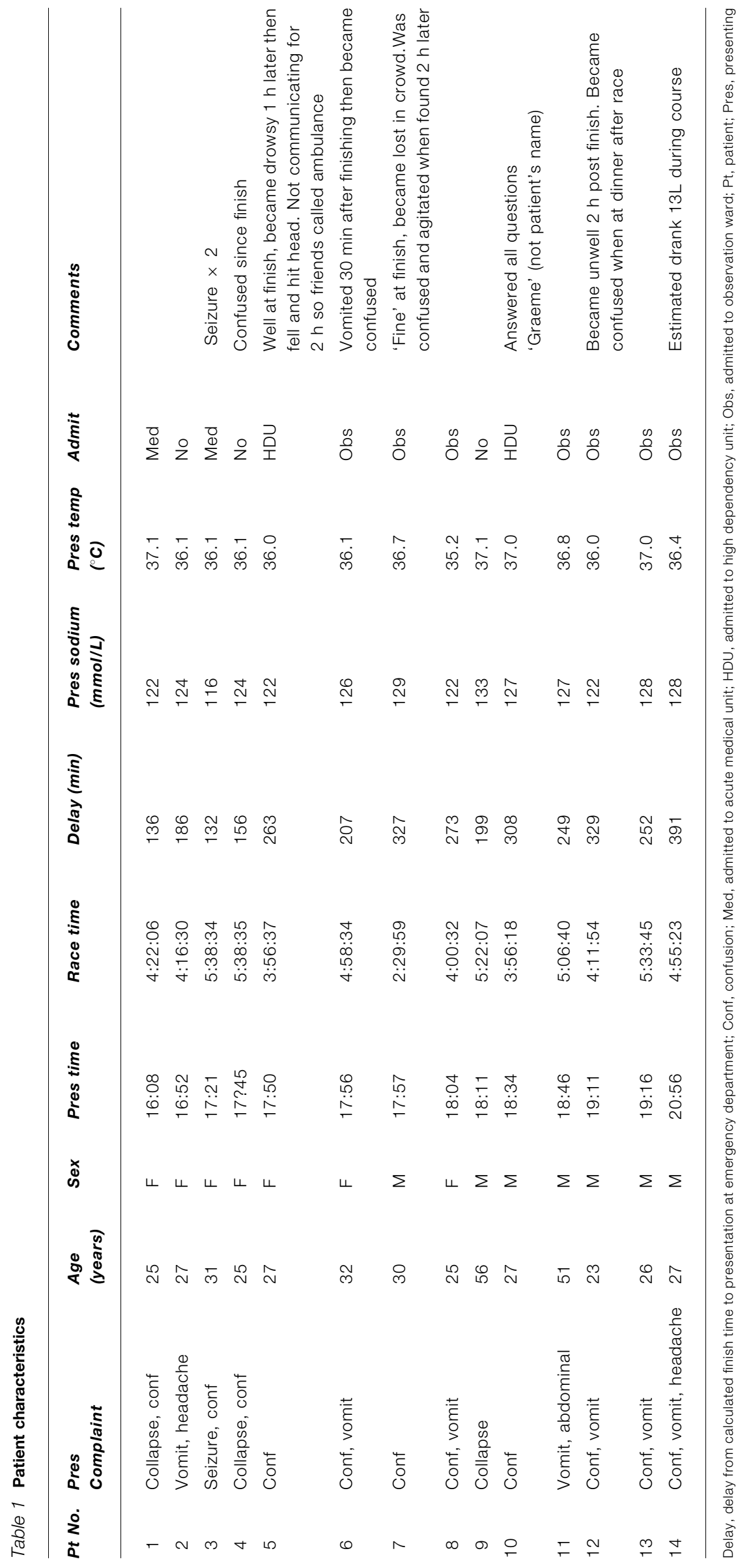




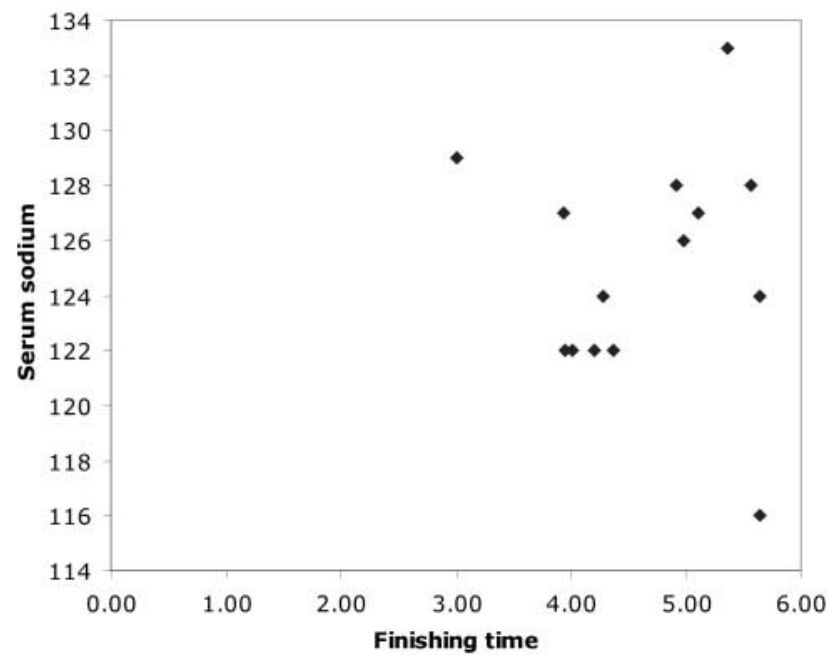

Figure 1 Graph finishing time v sodium

he had taken to complete the marathon. Information on NSAID use was not collected.

Attempts at follow up were made at 12 months. Relatives of four patients reported no ongoing problems, and the registered general practitioners of two reported no presentations to the practice and no correspondence from any other health facility indicating further problems.

\section{DISCUSSION}

In the London Marathon (1981-current), single, sporadic hospital cases of EAH, all of whom presented with seizures, were recorded in 1985, 1990, 1995 and 1996 (DS TunstallPedoe, personal records). No previous cluster of cases had occurred. We postulate that this was due to the higher than usual ambient temperature combined with widespread promotion of drinking to prevent dehydration and insufficient emphasis in the medical advice given to the runners on the risks of overhydration. A strong educational campaign including advice on safe drinking plus cooler atmospheric conditions were associated with only single reported hospital cases of EAH in 2004 and 2005. ${ }^{2}$ A copy of the advice provided to runners as part of their final instructions in 2004 and 2005 is given in the Box 1.

EAH is a dilutional hyponatraemia due to excess free water ingestion. ${ }^{1,4,5}$ Water and hypotonic fluids were freely available during the 2003 London Marathon. Our experience is consistent with previous reports that describe the condition occurring more commonly in women; but, unlike previous reports, we found no correlation with slow running time. We did note a significant delay between finishing and presentation to the hospital, with anecdotal reports that there were often delays between finishing and development of symptoms. We postulate that this may be related to fluids consumed at or after the completion of the marathon, which may explain the lack of correlation with running times.

In exercise associated hyponatraemia it is assumed that the serum sodium was normal prior to the event and that the hyponatraemia is acute, allowing rapid correction without the risk of demyelination. ${ }^{4}$ The treatment regime used in our patients was more conservative than recommended by some authors, ${ }^{6,7}$ resulting in rises in serum sodium over $4 \mathrm{~h}$ from -0.25 to $2 \mathrm{mmol} / \mathrm{L} / \mathrm{h}$ (mean $1.24 \mathrm{mmol} / \mathrm{L} / \mathrm{h}$ ). Despite receiving relatively hypertonic fluids, two patients demonstrated transient falls in their serum sodium concentrations. We postulate that this was due to continuing absorption of hypotonic fluids and ongoing renal sodium loss in response to hypervolaemia.

The neurohumeral response to exercise is complicated with changes in circulating volume, arginine vasopressin, atrial natriuretic peptide (ANP) and aldosterone being described. ${ }^{1,17,18}$ A study comparing renal handling of a free water load at rest found no difference in those subjects who had suffered EAH compared to control subjects who had not. ${ }^{19}$ Despite this, individual variations in urine output and sodium loss, possibly related to hypervolaemia and high ANP, may have contributed to the differences seen. Simple formulae to calculate the required sodium requirements may therefore not accurately predict changes in serum sodium. ${ }^{11}$

Table 2 Sodium versus fluid for hypertonic saline pts

\begin{tabular}{|c|c|c|c|c|c|c|c|c|}
\hline$P t$ & Sex & Initial $\mathrm{Na}^{+}$ & $0.9 \%$ Saline & $1.8 \%$ Saline & Time (h) & Repeat $\mathrm{Na}$ & $\begin{array}{l}\Delta \mathrm{Na} \\
\mathrm{mmol} / \mathrm{L} / \mathrm{H}\end{array}$ & $\begin{array}{l}\text { [Na] } \\
\text { following } \\
\text { morning } \\
\text { mmol/L }\end{array}$ \\
\hline
\end{tabular}

\begin{tabular}{rrrllllll}
\hline 1 & $F$ & 122 & 2000 & 250 & 3 & 126 & 1.3 & 136 \\
3 & $F$ & 116 & 1000 & 500 & 2 & 121 & 2.5 & 137 \\
5 & $F$ & 122 & 2000 & 500 & 3.5 & 126 & 1.1 & 132 \\
8 & $F$ & 122 & 2000 & 500 & 4 & 121 & -0.25 & 130 \\
10 & $M$ & 127 & 1000 & 1000 & 2 & 129 & 1.0 & 139 \\
12 & $M$ & 122 & 2500 & 1000 & 3.7 & 128 & 1.8 & 138 \\
\hline
\end{tabular}




\section{Box 1 Advice given to runners regarding drinking in 2004 and 2005}

\begin{abstract}
Drinking safely: a balancing act
Drinking too little can lead to problems, as you need to replace some of the fluid you lose as sweat. Drinking much too much can be very dangerous and lead to hyponatraemia (water intoxication), fits and even death. There were 15 serious cases in 2003 , luckily none of them fatal but all requiring hospital treatment. They had drunk far more than they needed and when they started feeling unwell thought they were short on water and drank even more.
\end{abstract}

Start the race well hydrated (pale looking urine) and drink when you can, especially in the first half of the race. This will help you feel better late in the race and may prevent cramp.

Do not gulp large volumes of salt free fluids (e.g. water) before, during or after the race. It is possible to become ill from drinking too much, too quickly; this condition (hyponatraemia) is becoming increasingly common in slower runners.

\section{A rough drinking guide}

Faster runners ( under $3 \mathrm{~h} 30 \mathrm{~min}$ ) on a warm day may need as much as a litre of fluid per hour (i.e. 2 pints). Slower runners should need less, particularly on a cool day, and should not drink more than a pint or so- $500 \mathrm{~mL} /$ hour. There are frequent water stations you do not need to drink at each one, just swallow a mouthful of water occasionally. If you like the Lucozade sport, drink that as well.

After the finish do not drink large amounts of water. You can only rehydrate gradually over the next $24 \mathrm{~h}$. Eat some salty food and space your drinks and you will not get hyponatraemia.

Despite the relatively slow correction of hyponatraemia in our patients, none developed any further complications and all made a complete recovery allowing rapid discharge. Although follow-up data were scant, we received no reports of any late complications in this group. Earlier and more aggressive use of hypertonic fluids may have prevented the drop in serum sodium seen in some of our patients and could reduce the risk of deterioration after presentation that has been described by other authors. ${ }^{7,17} \mathrm{We}$ would therefore advise earlier institution of hypertonic fluids than we used in this cohort.

\section{CONCLUSION}

Presentation of exercise associated hyponatraemia may be delayed and clinical deterioration after presentation has been described. Treatment is controversial but the use of isotonic $(0.9 \%)$ saline may not result in rises of serum sodium - possibly due to ongoing absorption of hypotonic fluids and ongoing sodium loss. Pending a randomized controlled trial of isotonic versus hypertonic fluid, we would suggest the early use of hypertonic fluids, noting that the optimum rate of serum sodium correction is still controversial. We would concur with other authors that mild cases can be managed expectantly without fluids (awaiting spontaneous diuresis) but more symptomatic patients require a more aggressive initial treatment regime including hypertonic saline. ${ }^{1,4,6,11}$

Competing interests None declared.

\section{REFERENCES}

1 Hew-Butler T, Almond C, Ayus JC, et al. Consensus statement of the 1st International Exercise-Associated Hyponatraemia Consensus Development Conference, Cape Town, South Africa 2005. Clin J Sport Med 2005; 15:208-13

2 Goudie AM, Tunstall-Pedoe DS, Kerins M. Altered mental status after a marathon. N Engl J Med 2005;352:1613-4

3 Tunstall-Pedoe DS. Marathon Myths and Marathon Medicine. In: TunstallPedoe DS, ed. Marathon Medicine. London: RSM Press Ltd, 2000:3-14

4 Speedy DB, Noakes TD, Schneider C. Exercise-associated hyponatraemia: a review. Emerg Med (Fremantle) 2001;13:17-27

5 Almond CS, Shin AY, Fortescue EB, et al. Hyponatraemia among runners in the Boston Marathon. N Engl J Med 2005;352:1550-6

6 Ayus JC, Varon J, Arieff AI. Hyponatraemia, cerebral edema, and noncardiogenic pulmonary edema in marathon runners. Ann Intern Med 2000;132:711-14

7 Davis DP, Videen JS, Marino A, et al. Exercise-associated hyponatraemia in marathon runners: a two-year experience. J Emerg Med 2001;21:47-57

8 Hew TD, Chorley JN, Cianca JC, Divine JG. The incidence, risk factors, and clinical manifestations of hyponatraemia in marathon runners. Clin J Sport Med 2003;13:41-7

9 Noakes TD, Sharwood K, Collins M, Perkins D. The dipsomania of great distance water intoxication in an ironman triathlete. Br J Sports Med 2004;38:E16

10 Adrogue HJ, Madias NE. Hyponatraemia. $N$ Engl J Med 2000; 342:1581-9

11 Moritz ML, Ayus JC. The pathophysiology and treatment of hyponatraemic encephalopathy: an update. Nephrol Dial Transplant 2003; 18:2486-91

12 Musch W, Xhaet O, Decaux G. Solute loss plays a major role in polydipsia-related hyponatraemia of both water drinkers and beer drinkers. QJ Med 2003;96:421-6

13 Hartung TK, Schofield E, Short AI, Parr MJ, Henry JA. Hyponatraemic states following 3,4-methylenedioxymethamphetamine (MDMA, 'ecstasy') ingestion. QJ Med 2002;95:431-7

14 Worthley LI, Thomas PD. Treatment of hyponatraemic seizures with intravenous 29.2\% saline. BMJ (Clin Res Ed) 1986;292:168-70

15 Zelingher J, Putterman C, Ilan Y, et al. Case series: hyponatraemia associated with moderate exercise. Am J Med Sci 1996;311:86-91

16 Lucozade Sport [http://www.lucozadesport.com] Accessed 3 April 2006

17 Irving RA, Noakes TD, Buck R, et al. Evaluation of renal function and fluid homeostasis during recovery from exercise-induced hyponatraemia. J Appl Physiol 1991;70:342-8

18 Speedy DB, Rogers IR, Noakes TD, et al. Exercise-induced hyponatraemia in ultradistance triathletes is caused by inappropriate fluid retention. Clin J Sport Med 2000;10:272-8

19 Speedy DB, Noakes TD, Boswell T, Thompson JM, Rehrer N, Boswell DR. Response to a fluid load in athletes with a history of exercise induced hyponatraemia. Med Sci Sports Exerc 2001;33:1434-42 